\title{
Nonlinear Turbulent Two-Dimensional Shallow-Water Equations And Their Numerical Solution
}

\author{
SUJIT K. BOSE
}

\author{
S.N. Bose National Centre for Basic Sciences, Kolkata 700106, West Bengal, INDIA.
}

Abstract:- Free surface flow of water over a shallow rough bed is characteristically turbulent due to disturbances generated by the bed resistance and diverse causes. The paper presents a derivation of the basic equations in two dimensions and their numerical solution by an extension of the method developed earlier for flow in one dimension. Starting from the three dimensional Reynolds Averaged Navier Stokes (RANS) equations, the equations of continuity and horizontal momenta are depth averaged to derive three equations for the free surface elevation $\zeta$ and the horizontal, depth averaged velocity components $(U, V)$. Certain closure assumptions are required for derivation of the equations. Principally, the viscous stresses are neglected, while the Reynolds stresses are assumed to depend on the vertical coordinate $z$ only for the shearing flow over the $x, y$ - plane representing the plane bed. Secondly, it is assumed that the instantaneous horizontal components of velocity $(u, v)$ follow the $1 / p$ th $(p=7)$ power law of variation in the $z$ - direction, in liu of the the logarithmic law of the wall. For numerical solution of the three nonlinear equations of continuity and momenta, the equations are reformulated in terms of the primitive "discharge" components $(Q, R)$ of the velocity $(U, V)$, showing that $Q$ and $R$ can be functions of $\zeta$ alone. The transformed equation of continuity is treated by the Lax-Richtmyer method. The two momentum equations on the other hand, transform in to two coupled second degree equations in the derivatives of $Q$ and $R$, which decouple in the important case of quasilinear straight crested waves on the water surface. The decoupled equations are numerically solved by the iterative modified Euler method, and illustrated by application to an initial elevation of a model for tsunami propagation.

Keywords: Two-dimensional shallow-water equations, Turbulence, RANS equations, Depth averaging, Discharge, Lax-Richtmyer method, Modified Euler Method.

Received: May 30, 2021. Revised: November 28, 2021. Accepted: December 22, 2021. Published: January 19, 2022.

\section{Introduction}

Shallow water equations in two dimensions are useful in oceanic and atmospheric flows as the aspect ratio of the horizontal spread to vertical height is very large. In the development of the equations, for oceanic flows such as tsunamis, tides, storm surges and oceanic circulations, the fluid pressure at a point is considered hydrostatic, even though the free surface may have vertical motion. The governing equations of mass conservation and the horizontal components of momentum are formally in terms of the surface elevation $\zeta$ and the depth averaged components of the horizontal velocity $(U, V)$. The equations neglecting the wind forses on the free surface of the water are (Mader [1], p. 27), the equation of continuity

$$
\frac{\partial \zeta}{\partial t}+\frac{\partial(\zeta U)}{\partial x}+\frac{\partial(\zeta V)}{\partial y}=0
$$

and the momentum equations in the $x, y$ - directions as

$$
\begin{aligned}
& \frac{\partial U}{\partial t}+U \frac{\partial U}{\partial x}+V \frac{\partial U}{\partial y}+g \frac{\partial \zeta}{\partial x}+\tau_{0 x}=0 \\
& \frac{\partial V}{\partial t}+U \frac{\partial V}{\partial x}+V \frac{\partial V}{\partial y}+g \frac{\partial \zeta}{\partial y}+\tau_{0 y}=0
\end{aligned}
$$

where $\left(\tau_{0 x}, \tau_{0 y}\right)$ are the components of bed resistance that are usually modeled in the hydraulics 
literature by either the Chezy or by the Manning empirical formulae of the following forms:

$$
\begin{gathered}
\tau_{0 x}=\frac{g U \sqrt{U^{2}+V^{2}}}{C^{2} \zeta}, \tau_{0 y}=\frac{g V \sqrt{U^{2}+V^{2}}}{C^{2} \zeta}, \quad \begin{array}{c}
(\text { Chezy) } \\
\tau_{0 x}=\frac{m^{2} g U \sqrt{U^{2}+V^{2}}}{\zeta^{1 / 3}}, \tau_{0 y}=\frac{m^{2} g V \sqrt{U^{2}+V^{2}}}{\zeta^{1 / 3}} \\
\text { (Manning) }(4 b)
\end{array}
\end{gathered}
$$

where $C$ and $m$ are respectively the Chezy and the Manning coefficients that depend on the roughnes of the bed, and $g$ the acceleration due to gravity The nonlinear partial differential equations (1) (3) are also treated by Mader [1] using first order finite differencing and applying them to a number of applications, in particular to tsunamis.

Among the more advanced methods, Fennema and Chowdhry [2], [3] have respectively given implicit and explicit type of finite difference methods. The former is of Beam-Warming type and the latter of MacCormack-Gobuti type, both being second order accurate in space as well as time. On the other hand, Wilders et. al. [4] and Casulli [5] develop fully implicit splitting and semi-implicit finite difference methods for the equations. In recent years, Ouyang et. al [6] present a MacCormackTVD finite difference scheme for solution, where as Fu and Hodges [7] present a time-centered splitimplicit method, citing some other works. Much earlier however, Katopodes and Strelkoff [8] treat the equations differently by the method of characteristic surfaces. Finite volume methods have also been proposed. Alcrudo and Garcia-Navarro [9] give a high resolution Godunov type scheme, while Peng and Tang [10] present a method for which a Godunov type scheme is used for solving the Riemann problem. Garcia-Navarro et. al. [11] have also presented a multidimensional upwind scheme. Oceanic circulation models based on the two dimensional shallow water equations are also commercially available as software packages.

Here, in this paper, the shallow water equations in two-dimensions are developed taking in to consideration the turbulence generated by bed friction and other causes. The methodology is a generalization of the one given by Bose and Dey [12] for the case of one dimensional open channel flows. Beginning with the three dimensional RANS equations, it is assumed that the Reynolds stress components at a point depend on the vertical $z$ coordinate of the point only in a flow, which is essentially of shearing type parallel to the bed. It is then argued following Schlichting [13] that the horizontal components of the time averaged instantaneous velocity $(u, v)$ vary as $z^{1 / p}$ where $p=7$. The proportionality constants appearing in the expressions for $u$ and $v$ are then written in terms of the respective depth-averaged horizontal velocities $(U, V)$, which are functions of the horizontal coordinates $(x, y)$ only. The one-seventh power law is used here instead of the better known logarithmic law of the wall, as the former leads to explicit depth averaged equations for the flow. An expression for the time averaged vertical velocity $w$ in terms of $U, V$ and $z$ follows from the equation of continuity. Similarly, depth averaging of the vertical component of the momentum equation subject to the assumption of linear variation of the stream surface with respect to $z$ yields the expression for the time averaged pressure $\bar{p}$, which contains apart from the hydrostatic pressure, nonlinear terms on account of vertical instantaneous and convective accelerations. Depth averaging of the continuity and the two horizontal momentum equations then yield the depth averaged continuity and momentum equations in terms of $\zeta, U$ and $V$. Numerical treatment of the nonlinear equations is then developed generalizing that given by Bose (2018) for one dimensional flows. Expressing the three equations in terms of "discharge" components $Q=\zeta U$ and $R=\zeta V$ parallel to the $x$ and $y$ axes, it is shown that the two quantities can be functions of $\zeta$ alone, that is to say, $Q=F(\zeta)$ and $R=G(\zeta)$. The two nonlinear momentum equations then reduce to two coupled second degree equations in $F^{\prime}(\zeta)$ and $G^{\prime}(\zeta)$, where the prime denotes differentiation with respect to $\zeta$. In the important case of quasi-linear nearly straight crested waves parallel to the $y$-axis, the equations decouple in to a quadratic equation in $F^{\prime}(\zeta)$ and an explicit equation for $G^{\prime}(\zeta)$. The averaged continuity equation on the other hand reduces to a standard form conservation equation, which is treated by the second 
order Lax-Richtmyer method [16], where in the first order pair of ordinary differential equations fot $F(\zeta)$ and $G(\zeta)$ is solved by the second order iterative modified Euler method (Bose [15]. The method is illustrated by application to an initial elevation form proposed in Howe [17], for a model tsunami propagation problem.

\section{The Turbulent 2D Shallow Water Equa- tions}

The two-dimensional turbulent shallow-water freesurface flow over a plane horizontal bed is governed by the general RANS equations. As the free surface in general, can be undulating during the flow, the plane bed is conveniently chosen as the $x, y$-plane with the $z$-axis drawn vertically upwards, with a mean water depth of $h$ units. Due to turbulence, the velocity at a point $(x, y, z)$ in the fluid consists of the time averaged components $(u, v, w)$ and instantaneous fluctuations $\left(u^{\prime}, v^{\prime}, w^{\prime}\right)$. The time averaged components satisfy the RANS equation of continuity

$$
\frac{\partial u}{\partial x}+\frac{\partial v}{\partial y}+\frac{\partial w}{\partial z}=0
$$

for incompressible fluids. The fluctuating velocity components satisfy a similar equation. The RANS momentum equations (Schlichting [13], p. $562)$ contain contributions of $(u, v, w)$, that of the pressure $\bar{p}$, and the viscous and Reynolds stresses. Moreover, as the motion over the horizontal bed is essentially of shearing type, the viscous and Reynolds stress components become practically independent of $x$ and $y$. Hence, neglecting the contribution of the normal viscous stress in the vertical direction, the RANS momentum equations read as

$\frac{\partial u}{\partial t}+u \frac{\partial u}{\partial x}+v \frac{\partial u}{\partial y}+w \frac{\partial u}{\partial z}=-\frac{1}{\rho} \frac{\partial \bar{p}}{\partial x}+\frac{1}{\rho} \frac{\partial \tau_{z x}}{\partial z}+\frac{\mu}{\rho} \frac{\partial^{2} u}{\partial z^{2}}$

$\frac{\partial v}{\partial t}+u \frac{\partial v}{\partial x}+v \frac{\partial v}{\partial y}+w \frac{\partial v}{\partial z}=-\frac{1}{\rho} \frac{\partial \bar{p}}{\partial y}+\frac{1}{\rho} \frac{\partial \tau_{z y}}{\partial z}+\frac{\mu}{\rho} \frac{\partial^{2} v}{\partial z^{2}}$

$\frac{\partial w}{\partial t}+u \frac{\partial w}{\partial x}+v \frac{\partial w}{\partial y}+w \frac{\partial w}{\partial z}=-g-\frac{1}{\rho} \frac{\partial \bar{p}}{\partial z}-\frac{\partial \overline{w^{\prime 2}}}{\partial z}$

where $\rho=$ density, $\mu=$ dynamical coefficient of viscosity, $g=$ acceleration due to gravity, and $\tau_{z x}=-\rho \overline{w^{\prime} u^{\prime}}, \tau_{z y}=-\rho \overline{w^{\prime} v^{\prime}}$ are Reynolds stresses in which the overbars denote time averages. The Eqs. (5) - (8) describing the flow is under determined, and so additional conditions have to be incorporated.

\section{The $1 / \mathrm{p}^{\text {th }}$ Power Law}

For open channel flow in one direction only, say the $x$-direction, it is known that $u$ varies slowly as $z^{1 / p}$, where $p=7$ (Schlichting [13], p. 590; Bose and Dey [12]). Similarly, in the present generalization, one can assume that

$$
\begin{aligned}
u=\frac{p+1}{p} U(x, y, t) & \left(\frac{z}{\zeta}\right)^{1 / p}, \\
v & =\frac{p+1}{p} V(x, y, t)\left(\frac{z}{\zeta}\right)^{1 / p}
\end{aligned}
$$

where the velocity $(U, V)$ equals the depth average of $(u, v)$, and $\zeta(x, y)$ is the surface elevation over the point $(x, y)$ on the bed. Accordingly, from Eq. (5) by integration

$$
w=-\int_{0}^{z}\left(\frac{\partial u}{\partial x}+\frac{\partial v}{\partial y}\right) d z=-\left(\frac{\partial U}{\partial x}+\frac{\partial V}{\partial y}\right) \frac{z^{1 / p+1}}{\zeta^{1 / p}}
$$

where it is assumed for simplicity that the forward flux $(\partial U / \partial x, \partial V / \partial y)$ of the flow is much greater than the slope factor $(U / \zeta)(\partial \zeta / \partial x, \partial \zeta / \partial y)$ of the free surface, so that the latter terms become insignificant compared to the term written in equation (10). Physically, the approxiation (10) implies that as the rate of expansion $\partial U / \partial x+\partial V / \partial V$ incrases the vertical velocity $w$ decreases, which apparently is quite plausible due to the mass conservation principle. The solution forms (9) and (10) are next used in equations (5) - (8). The convective acceleration term in Eq. (8), using Eq. (5) becomes

$$
\begin{aligned}
& u \frac{\partial w}{\partial x}+v \frac{\partial w}{\partial y}+w \frac{\partial w}{\partial z}=u \frac{\partial w}{\partial x}+v \frac{\partial w}{\partial y}-w\left(\frac{\partial u}{\partial x}+\frac{\partial v}{\partial y}\right) \\
& =u^{2} \frac{\partial}{\partial x}\left(\frac{w}{u}\right)+v^{2} \frac{\partial}{\partial y}\left(\frac{w}{v}\right)=u^{2} \frac{\partial^{2} z_{1}}{\partial x^{2}}+v^{2} \frac{\partial^{2} z_{1}}{\partial y^{2}}
\end{aligned}
$$

wherein $z=z_{1}(x, y)$ represents the stream surface passing through the point $(x, y, z)$. The two partial derivatives $\partial^{2} z_{1} / \partial x^{2}$ and $\partial^{2} z_{1} / \partial y^{2}$ in equation (11) are proportional to the curvature of the surface $z=z_{1}(x, y)$ and is assumed to linearly vary 
with $z$, as in the Boussinesq approximation of open where $\alpha=(p+1)^{2} /[p(p+2)]$. On the other hand, channel flows. Then,

$$
\frac{\partial^{2} z_{1}}{\partial x^{2}}=\frac{z}{\zeta} \frac{\partial^{2} \zeta}{\partial x^{2}}, \quad \frac{\partial^{2} z_{1}}{\partial y^{2}}=\frac{z}{\zeta} \frac{\partial^{2} \zeta}{\partial y^{2}}
$$

Hence using Eq. (9), the convective acceleration (11) becomes

$$
\begin{aligned}
& u \frac{\partial w}{\partial x}+v \frac{\partial w}{\partial y}+w \frac{\partial w}{\partial z} \\
& =\left(\frac{p+1}{p}\right)^{2}\left(\frac{z}{\zeta}\right)^{2 / p+1}\left(U^{2} \frac{\partial^{2} \zeta}{\partial x^{2}}+V^{2} \frac{\partial^{2} \zeta}{\partial y^{2}}\right)
\end{aligned}
$$

Inserting the expression (13) in equation (8) and integrating over depth, one gets

$$
\begin{aligned}
& \frac{p}{\rho}=-\int_{\zeta}^{z} \frac{\partial w}{\partial t} d z-\frac{p+1}{2 p} \zeta\left\{\left(\frac{z}{\zeta}\right)^{2 / p+2}-1\right\} \\
& \times\left(U^{2} \frac{\partial^{2} \zeta}{\partial x^{2}}+V^{2} \frac{\partial^{2} \zeta}{\partial y^{2}}\right)-\overline{w^{2}}-g(z-\zeta)+\frac{\bar{p}_{0}}{\rho}
\end{aligned}
$$

where $\bar{p}_{0}$ is the constant pressure over the free surface $z=\zeta$. Thus the formal expression for the pressure is also obtained. In equation (14), the last two terms represent the ontribution of hydrostatic pressure and the rest represent the additional contribution due to turbulence.

\section{Depth Averaging}

As the water depth is shallow, it is appropriate to consider the depth averages of the continuity and horizontal momentum equations (5) - (7). Integration of equation (5) with respect to $z$ over $(0, \zeta)$ yields the depth averaged continuity equation (1). Treating the momentum equations (6) and (7) in a similar manner, the former leads to the equation

$$
\begin{gathered}
\frac{\partial}{\partial t}(\zeta U)+\frac{\partial}{\partial x}\left(\int_{0}^{\zeta} u^{2} d z\right)+\frac{\partial}{\partial y}\left(\int_{0}^{\zeta} u v d z\right) \\
+\frac{1}{\rho} \int_{0}^{\zeta} \frac{\partial \bar{p}}{\partial x} d z+\frac{\tau_{0 x}}{\rho}=0
\end{gathered}
$$

where $\tau_{0 x}=$ bed resistance parallel to the $x$-axis. The representations (9) yield for the first two integrals of equation (15) as

$$
\int_{0}^{\zeta} u^{2} d z=\alpha \zeta U^{2}, \quad \int_{0}^{\zeta} u v d z=\alpha \zeta U V
$$

as $\bar{p}(x, y, \zeta)=0$ on the free surface. Hence using Eq. (10) with Eqs. (5),

$$
\begin{aligned}
\frac{1}{\rho} \int_{0}^{\zeta} \frac{\partial \bar{p}}{\partial x} d z & =\gamma \frac{\partial}{\partial x}\left[\zeta^{2}\left(U^{2} \frac{\partial^{2} \zeta}{\partial x^{2}}+V^{2} \frac{\partial^{2} \zeta}{\partial y^{2}}\right)\right] \\
& -\delta \frac{\partial}{\partial x}\left[\zeta^{3} \frac{\partial}{\partial t}\left(\frac{\partial U}{\partial x}+\frac{\partial V}{\partial y}\right)\right. \\
& \left.-\frac{\zeta^{3}}{p} \frac{\partial \zeta}{\partial t}\left(\frac{\partial U}{\partial x}+\frac{\partial V}{\partial y}\right)\right]+g \frac{\partial \zeta}{\partial x}
\end{aligned}
$$

in which, $\gamma=(p+1)^{2} /[p(3 p+2)], \delta=p /(3 p+1)$, and the Reynolds normal stress term $\partial\left(\overline{w^{\prime 2}}\right) / \partial x$ is negligiible as assumed earlier. The contribution of the bed shear term $\tau_{0 x}=-\mu(\partial u / \partial z)_{z=0}$ in equation (15) can only be estimated. Here the Manning's formula (4b) is adopted as the coefficient $m$ appearing in the formula has lesser variation with respect to variation with the roughness of the bed surface. Equation (15) therefore becomes,

$$
\begin{gathered}
\frac{\partial}{\partial t}(\zeta U)+\alpha \frac{\partial}{\partial x}\left(\zeta U^{2}\right)+\alpha \frac{\partial}{\partial y}(\zeta U V) \\
+\gamma \frac{\partial}{\partial x}\left[\zeta^{2}\left(U^{2} \frac{\partial^{2} \zeta}{\partial x^{2}}+V^{2} \frac{\partial^{2} \zeta}{\partial y^{2}}\right)\right] \\
-\delta \frac{\partial}{\partial x}\left[\zeta^{3} \frac{\partial}{\partial t}\left(\frac{\partial U}{\partial x}+\frac{\partial V}{\partial y}\right)-\frac{\zeta^{2}}{p} \frac{\partial \zeta}{\partial t}\left(\frac{\partial U}{\partial x}+\frac{\partial V}{\partial y}\right)\right] \\
+g \zeta \frac{\partial \zeta}{\partial x}+\frac{m^{2} g U \sqrt{U^{2}+V^{2}}}{\zeta^{1 / 3}}=0
\end{gathered}
$$

In a similar manner Eq. (7) leads to the averaged equation

$$
\begin{gathered}
\frac{\partial}{\partial t}(\zeta V)+\alpha \frac{\partial}{\partial y}\left(\zeta V^{2}\right)+\alpha \frac{\partial}{\partial x}(\zeta U V) \\
+\gamma \frac{\partial}{\partial y}\left[\zeta^{2}\left(U^{2} \frac{\partial^{2} \zeta}{\partial x^{2}}+V^{2} \frac{\partial^{2} \zeta}{\partial y^{2}}\right)\right]- \\
\delta \frac{\partial}{\partial y}\left[\zeta^{3} \frac{\partial}{\partial t}\left(\frac{\partial U}{\partial x}+\frac{\partial V}{\partial y}\right)-\frac{\zeta^{2}}{p} \frac{\partial \zeta}{\partial t}\left(\frac{\partial U}{\partial x}+\frac{\partial V}{\partial y}\right)\right] \\
+g \zeta \frac{\partial \zeta}{\partial y}+\frac{m^{2} g V \sqrt{U^{2}+V^{2}}}{\zeta^{1 / 3}}=0
\end{gathered}
$$

Equations (1), (19) and (20) are then the depth averaged continuity and the $x, y$ components of 
WSEAS TRANSACTIONS on HEAT and MASS TRANSFER DOI: $10.37394 / 232012.2022 .17 .5$

the momentum equation.

\section{Simplification for Numerical Solution}

Since $p=7$ approximately, $\alpha=64 / 63 \approx$ $1.01587 \cdots \approx 1, \gamma=64 / 161=0.39751 \cdots \approx 0.4=$ $2 / 5$, and $\delta=7 / 22$. The approximation for $\alpha$ leads to familiar convective acceleration terms of equations (2) and (3), as can be seen in the equations (23) and (24) derived in the following. The approximation for $\gamma$ is introduced to bring some elegance to the basic equations. The highly nonlinear fifth term in both of the equations (19) and (20) arise due to the instantaneous vertical acceleratio $\partial w / \partial t$. For developing the numerical solution method, it is first noted that the two terms are horizontal gradients that need not be large. Hence, one obtains by iteration of (19) and (20), the first order approximations

$$
\begin{gathered}
\frac{\partial U}{\partial t} \approx-g \frac{\partial \zeta}{\partial x}, \quad \frac{\partial V}{\partial t} \approx-g \frac{\partial \zeta}{\partial y} \text { and } \\
\frac{\partial U}{\partial x}+\frac{\partial V}{\partial y} \approx-\frac{1}{\zeta} \frac{\partial \zeta}{\partial t}
\end{gathered}
$$

The left hand side quantities of (21) are then inserted in the $\delta$-terms of equations (19) and (20). Thus equation (19) becomes

$$
\begin{gathered}
\zeta \frac{\partial U}{\partial t}-\zeta U\left(\frac{\partial U}{\partial x}+\frac{\partial V}{\partial y}\right)+\frac{\partial}{\partial x}\left[\zeta\left\{U^{2}-\frac{1}{22}\left(\frac{\partial \zeta}{\partial t}\right)^{2}\right\}\right] \\
+\frac{\partial}{\partial y}(\zeta U V)+\frac{2}{5} \frac{\partial}{\partial x}\left[\zeta^{2}\left(U^{2} \frac{\partial^{2} \zeta}{\partial x^{2}}+V^{2} \frac{\partial^{2} \zeta}{\partial y^{2}}\right)\right] \\
+\frac{7 g}{22} \frac{\partial}{\partial x}\left[\zeta^{3}\left(\frac{\partial^{2} \zeta}{\partial x^{2}}+\frac{\partial^{2} \zeta}{\partial y^{2}}\right)\right]+g \zeta \frac{\partial \zeta}{\partial x} \\
+\frac{m^{2} g U \sqrt{U^{2}+V^{2}}}{\zeta^{1 / 3}}=0
\end{gathered}
$$

A further approximation is possible in equation (22) if one asssumes that $U^{2}>>\frac{1}{22}\left(\frac{\partial \zeta}{\partial t}\right)^{2}$, as the horizontal velocity is much larger than the instantaneous vertical velocity of the free surface. With this assumption, equation (22) simplifies to

$$
\frac{\partial U}{\partial t}+U \frac{\partial U}{\partial x}+V \frac{\partial U}{\partial y}+\frac{2}{5 \zeta} \frac{\partial}{\partial x}\left[\zeta^{2}\left(U^{2} \frac{\partial^{2} \zeta}{\partial x^{2}}+V^{2} \frac{\partial^{2} \zeta}{\partial y^{2}}\right)\right]
$$

$$
\begin{gathered}
+\frac{7 g}{22 \zeta} \frac{\partial}{\partial x}\left[\zeta^{3}\left(\frac{\partial^{2} \zeta}{\partial x^{2}}+\frac{\partial^{2} \zeta}{\partial y^{2}}\right)\right]+g \frac{\partial \zeta}{\partial x} \\
+\frac{m^{2} g U \sqrt{U^{2}+V^{2}}}{\zeta^{/ 3}}=0
\end{gathered}
$$$$
\text { Sujit K. Bose }
$$

Similarly equation (20) reduces to

$$
\begin{gathered}
\frac{\partial V}{\partial t}+U \frac{\partial V}{\partial x}+V \frac{\partial V}{\partial y}+\frac{2}{5 \zeta} \frac{\partial}{\partial y}\left[\zeta^{2}\left(U^{2} \frac{\partial^{2} \zeta}{\partial x^{2}}+V^{2} \frac{\partial^{2} \zeta}{\partial y^{2}}\right)\right] \\
+\frac{7 g}{22 \zeta} \frac{\partial}{\partial y}\left[\zeta^{3}\left(\frac{\partial^{2} \zeta}{\partial x^{2}}+\frac{\partial^{2} \zeta}{\partial y^{2}}\right)\right]+g \frac{\partial \zeta}{\partial y} \\
+\frac{m^{2} g V \sqrt{U^{2}+V^{2}}}{\zeta^{/ 3}}=0
\end{gathered}
$$

The continuity and momentum equations (1) and (23) - (24) are in physical dimensions. In order to nondimensionalisze the equations, let the variables $x, y, t, \zeta$ and $U, V$ be temporarily written as $\hat{x}, \hat{y}, \hat{t}, \hat{\zeta}$ and $\hat{U}, \hat{V}$ respectively; then writing

$$
\begin{gathered}
\hat{x}=h x, \hat{y}=h y, \hat{\zeta}=h \zeta, \hat{t}=t \sqrt{h / g}, \quad \text { and } \\
\hat{U}=U \sqrt{g h}, \hat{V}=V \sqrt{g h}
\end{gathered}
$$

in which $h$ is the mean depth of water; then the variables $x, y, \zeta, t$ and $U, V$ become nondimensional. By the transformations (25), equation (1) remains unchanged in form, while the factor $g$ drops out from the equations (23) and (24). Equation (23) with $V=0$ and $\partial(\cdot) / \partial y=0$ corresponds to the one dimensional case studied by Bose [14], but here it is slightly simpler in form.

\section{Transformation and Localization Assump- tion}

In order to develop the numerical procedure for solving the equations (1) and (23) - (24), it is noted that the continuity equation (1) relates the instantaneous vertical velocity of the free surface at $(x, y)$ to the gradient of the discharge $(Q, R)$ in the $x, y$ - directions defined by

$$
Q:=\zeta U, \quad R:=\zeta V
$$

so that equation (1) in compact substricted notoation for partial derivatives becomes

$$
\zeta_{t}+Q_{x}+R_{y}=0
$$


In terms of $Q, R$ one has

$$
\begin{aligned}
& U=\frac{Q}{\zeta}, \quad U_{t}=\frac{Q_{t}}{\zeta}+\frac{Q}{\zeta^{2}}\left(Q_{x}+R_{y}\right), \\
& U_{x}=\frac{Q_{x}}{\zeta}-\frac{Q}{\zeta^{2}} \zeta_{x}, \quad U_{y}=\frac{Q_{y}}{\zeta}-\frac{Q}{\zeta^{2}} \zeta_{y} \\
& V=\frac{R}{\zeta}, \quad V_{t}=\frac{R_{t}}{\zeta}+\frac{R}{\zeta^{2}}\left(Q_{x}+R_{y}\right), \\
& V_{x}=\frac{R_{x}}{\zeta}-\frac{R}{\zeta^{2}} \zeta_{x}, \quad V_{y}=\frac{R_{y}}{\zeta}-\frac{R}{\zeta^{2}} \zeta_{y}
\end{aligned}
$$

where in the second equations of (28) and (29), the transformed form of the continuity equation (27) is used. In terms of $Q$ and $R$ in place of $U$ and $V$ respectively, equations (23) and (24) become

$$
\begin{gathered}
\zeta^{2} Q_{t}+Q\left(2 \zeta Q_{x}-Q \zeta_{x}+\zeta R_{y}\right)+R\left(\zeta Q_{y}-Q \zeta_{y}\right) \\
+\frac{2}{5} \zeta^{2}\left(Q^{2} \zeta_{x x x}+R^{2} \zeta_{x y y}\right)+\frac{4}{5} \zeta^{2}\left(Q Q_{x} \zeta_{x x}+R R_{x} \zeta_{y y}\right) \\
+\frac{7}{22} \zeta^{4}\left[\zeta\left(\zeta_{x x x}+\zeta_{x y y}\right)+3 \zeta_{x}\left(\zeta_{x x}+\zeta_{y y}\right)\right] \\
+\zeta^{3} \zeta_{x}+\frac{m^{2} Q \sqrt{Q^{2}+R^{2}}}{\zeta^{1 / 3}}=0
\end{gathered}
$$

and

$$
\begin{gathered}
\zeta^{2} R_{t}+\zeta\left(R Q_{x}+Q R_{x}+2 R R_{y}\right)-R\left(Q \zeta_{x}+R \zeta_{y}\right) \\
+\frac{2}{5} \zeta^{2}\left(Q^{2} \zeta_{x x y}+R^{2} \zeta_{y y y}\right)+\frac{4}{5} \zeta^{2}\left(Q Q_{y} \zeta_{x x}+R R_{y} \zeta_{y y}\right) \\
+\frac{7}{22} \zeta^{4}\left[\zeta\left(\zeta_{x x y}+\zeta_{y y y}\right)+3 \zeta_{y}\left(\zeta_{x x}+\zeta_{y y}\right)\right]+ \\
\zeta^{3} \zeta_{y}+\frac{m^{2} R \sqrt{Q^{2}+R^{2}}}{\zeta^{1 / 3}}=0
\end{gathered}
$$

For numerically solving the partial differential equations (29) - (31) an invertibility assumption is now made as in Bose [14]. Since the solution of these equations is of the form $\zeta=\zeta(x, y, t), \quad Q=$ $Q(x, y, t), \quad R=R(x, y, t)$, if the inverse of these functions exist then, $x=x(\zeta, Q, R), y=$ $y(\zeta, Q, R), t=t(\zeta, Q, R)$. A sufficient condition for the inversion to be possible is that the Jacobian of the transformation does no vanish at points within the domain. Under this assumption, $Q=Q[x(\zeta, Q, R), y(\zeta, Q, R), t(\zeta, Q, R)]$ (a function of $\zeta, Q, R$ ) and similarly, $R=$
$R[x(\zeta, Q, R), y(\zeta, Q, R), t(\zeta, Q, R)]$ (also a function of $\zeta, Q, R)$. The fixed point solution of this pair iterative pair of equations, for each $\zeta$, by elimination of $R$ and $Q$ respectively, is of the form

$$
Q=F(\zeta), \quad R=G(\zeta)
$$

provided the functios $F$ and $G$ are Lipschitz continuous. Physically as was observed in Bose [14], equation (32) implies that the discharge components $Q$ and $R$ at a point $(x, y)$ and at time $t$ depends solely on the local elevation $\zeta$ at that point and time, as in the theory of long tidal waves. The numerical method developed here may therefore be considered as an extension of that theory to nonlinear turbulent waves. Substituting the forms (32) in equations (27) yields the linear conservation equation

$$
\zeta_{t}+F_{x}(\zeta)+G_{y}(\zeta)=0
$$

The same substitution in Eqs. (30), (31) however yields a pair of coupled second degree equations in the derivatives $F^{\prime}(\zeta)$ and $G^{\prime}(\zeta)$ that are difficult to solve algebraically.

In the important case of almost linear crests prpagating in the direction of the $x$-axis however, $\left|\zeta_{y}\right|<<1$ and $|G|<<|F|$ so that the two equations can be solved approximately. To the first order of $\zeta_{y}$ and $G$, one gets

$$
a F^{2}-b F F^{\prime}+c=0
$$

and

$$
\begin{gathered}
G^{\prime}=\frac{1}{\zeta_{x}\left(\zeta F^{\prime}-F\right)}\left[\zeta_{x} F^{\prime} G-\frac{\zeta_{x} F G}{\zeta}+\frac{2}{5} \zeta \zeta_{x x y}\right. \\
+\frac{4}{5} \zeta \zeta_{y} \zeta_{x x} F F^{\prime}+\frac{7}{22} \zeta^{3}\left\{\zeta\left(\zeta_{x x y}+\zeta_{y y y}\right)+3 \zeta_{x x} \zeta_{y}\right\} \\
+\zeta^{2} \zeta_{y}+\frac{m^{2} F G \operatorname{sgn}(F)}{\zeta^{4 / 3}}
\end{gathered}
$$

where

$$
\begin{aligned}
& a=\zeta^{2}, \quad b=2 \zeta\left(1+\frac{2}{5} \zeta \zeta_{x x}\right), \\
& c=\left(1-\frac{2}{5} \zeta^{2} \frac{\zeta_{x x x}}{\zeta_{x}}-\frac{m^{2} \operatorname{sgn}(F)}{\zeta_{x} \zeta^{1 / 3}}\right) F^{2} \\
& -\frac{7}{22} \zeta^{4}\left[\frac{\zeta}{\zeta_{x}}\left(\zeta_{x x x}+\zeta_{x y y}\right)+3\left(\zeta_{x x}+\zeta_{y y}\right)\right]-\zeta^{3}
\end{aligned}
$$

Equations (33) - (35) are now treated numerically. 


\section{The Numerical Procedure}

Since the discharge $Q$ or $F(\zeta)$ is an increasing function of $\zeta$, the appropriate solution of the quadratic equation (34) is

$$
F^{\prime}=\frac{b F+\sqrt{b^{2} F^{2}-4 a c}}{2 a}
$$

For real solution $b^{2} F^{2} \geq 4 a c$ representing undulating flow surface; otherwise $F^{\prime}=0$ for steady discharge. Eq. (37) is inegrated by the trapezoidal rule based iterative, second order, modified Euler method (Bose [15], p. 264). The coefficients $a, b, c$ given in equation (36) contain partial derivatives of $\zeta$ up to the third order partial derivatives. These derivatives are calculated by second order finite differences of $\zeta$ over a grid of points $x=(m-1) \Delta x, y=(n-1) \Delta y,(m=$ $1,2,3, \cdots ; n=1,2,3, \cdots)$, where $\Delta x, \Delta y$ are the grid lengths parallel to the $x$ and $y$ - axes respectively. The value of $F$ and $G$ at the grid points are then obtained by the modified Euler method by recursion as:

$$
\begin{aligned}
F(m+1, n) & =F(m, n)+\Delta x \times\left[F^{\prime}(m, n) \zeta_{x}(m, n)\right] \\
& \leftarrow \frac{1}{2}[F(m, n)+F(m+1, n)] \\
G(m, n+1) & =G(m, n)+\Delta y \times\left[G^{\prime}(m, n) \zeta_{y}(m, n)\right] \\
& \leftarrow \frac{1}{2}[G(m, n)+G(m, n+1)]
\end{aligned}
$$

Equations (38), (39) determine the values of the functions $F$ and $G$ for a given elevation $\zeta$ at a given time $t$. The evolution of $\zeta$ as solution of equation (33) is obtained by application of the second order Lax-Richtmyer scheme for increasing time $t=l \Delta t,(l=1,2,3, \cdots)$. According to Richtmyer and Morton [16], the time evolution of $\zeta$ from given step $l$ is obtained in two steps $l+1$ and $l+2$ :

$$
\zeta_{m, n}^{l+1}=\frac{1}{4}\left(\zeta_{m+1, n}^{l}+\zeta_{m-1, n}^{l}+\zeta_{m, n+1}^{l}+\zeta_{m, n-1}^{l}\right)
$$

$-\frac{\Delta t}{2 \Delta x}\left(F_{m+1, n}^{l}-F_{m-1, n}^{l}\right)-\frac{\Delta t}{2 \Delta y}\left(G_{m, n+1}^{l}-G_{m, n-1}^{l}\right)$ where $F_{m, n}^{l}=F\left(\zeta_{m, n}^{l}\right)$, and at the second step

$$
\begin{aligned}
\zeta_{m, n}^{l+2}=\zeta_{m, n}^{l} & -\frac{\Delta t}{\Delta x}\left(F_{m+1, n}^{l+1}-F_{m-1, n}^{l+1}\right) \\
& -\frac{\Delta t}{\Delta y}\left(G_{m, n+1}^{l+1}-G_{m, n-1}^{l+1}\right)
\end{aligned}
$$

Equation (41) determines the elevation at the next step. In practice one can take $\Delta x=\Delta y$ and $\Delta t=r \Delta x$, where the factor $r<1$ is so chosen that the computation remains stable and convergent for small changes in its value.

\section{Application to a model Initial Surface El- evation}

The numerical method of the preceding section is applied to a model initial surface elevation considered by Howe [17], p.332 for tsunami propagation caused by sudden uplifting of the sea bed in the form of a ridge caused by subduction of the bed in front. Accordingly the initial elevation of the sea surface is assumed to follow the dislocated bed form with a profile given by the equation

$$
\begin{aligned}
\zeta=1 & +\zeta_{0}\left[\frac{1}{\left\{1+\left[(x+L)^{2}+y^{2}\right] / L^{2}\right\}^{3 / 2}}\right. \\
& \left.-\frac{1}{\left\{1+\left[(x-L)^{2}+y^{2}\right] / L^{2}\right\}^{3 / 2}}\right]
\end{aligned}
$$

where $\zeta_{0}=$ maximum elevation/depression above/below the mean surface of water, and $2 \mathrm{~L}$ $=$ distance between the highest and the lowest ponts of the initial free surface. For presentable results it is assumed that $\zeta_{0}=0.3$ and $L=10$. The profile tapers to plane level $\zeta=1$ as $|y|$ tends to infinity. The initial value of $\zeta$ at $t=0$ is computed for $x, y \geq 0$ from Eq. (42), taking $\Delta x=\Delta y=0.1$ and $\Delta t=r \Delta x$, where $r=1 / 2$. The numerical scheme is found to yield stable and convergent values of $\zeta$ for small changes in the value of $r$. The initial value of the functions $F$ and $G$ are estimated from the following consideration. Since the crests are nearly parallel to the $y$-axis, $G$ is negligible. If moreover, the crests are assumed to move with a constant sub-critical velocity $c_{0}$ to avoid choking of flow, then following Eq. $(33), F^{\prime}(\zeta)=c_{0}$, so that $F(\zeta)=c_{0} \zeta$. Accoridngly a value of $c_{0}=0.2$ is assumed here to compute $F(\zeta)$ at the different grid points. The spatial derivatives of the function 
$\zeta$ are then computed by the second order finite difference formulae:

$$
\begin{gathered}
\zeta_{x}=\frac{\zeta(x+\Delta x, y)-\zeta(x-\Delta x, y)}{(\Delta x)^{2}} \\
\zeta_{x x}=\frac{\zeta(x+\Delta x, y)-2 \zeta(x, y)+\zeta(x-\Delta x, y)}{(\Delta x)^{2}} \\
\zeta_{x x x}(x, y)=\frac{1}{\Delta x^{3}}[\zeta(x+2 \Delta x, y)-3 \zeta(x+\Delta x, y) \\
+3 \zeta(x, y)-\zeta(x-\Delta x, y)] \quad(45) \\
\zeta_{x x y}(x, y)=\frac{1}{2(\Delta x)^{3}}[\zeta(x+\Delta x, y+\Delta y) \\
-\zeta(x+\Delta x, y-\Delta y)-2 \zeta(x, y+\Delta y)+2 \zeta(x, y-\Delta y) \\
+\zeta(x-\Delta x, y+\Delta y)-\zeta(x-\Delta x, y-\Delta y)] \quad(46)
\end{gathered}
$$

and similar expressions for $\zeta_{y}(x, y), \zeta_{y y}(x, y)$, $\zeta_{y y y}(x, y)$, and $\zeta_{x y y}(x, y)$, for $x, y>0$ or, $m, n \geq 2$. For $x=0$ and $y=0$, linear extrapolations

$$
\zeta_{x}(0, y)=2 \zeta_{x}(\Delta x, y)-\zeta_{x}(2 \Delta x, y)
$$

$$
\zeta_{x}(x, 0)=2 \zeta_{x}(x, \Delta y)-\zeta_{x}(x, 2 \Delta y)
$$

and similar expressions for the other partial derivatives are used. The time evolution of the elevation $\zeta$ following the Lax-Richtmyer scheme (40), (41) is then carried out using Eqs. (38), (39). The corresponding value of the discharge functions $F$ and $G$ is carried out as at the initial time, i.e. $F=c_{0} \zeta$ and $G=0$. Performing a large number of iterations over time, the computed results for $\zeta$ at times $t=0,20,40$ and 60 with respect to $x$ for the three representative cases $y=0, y=\frac{1}{2} x$, and $y=x$ are presented in the figures 1,2 , and 3 respectively. The three cases correspond to the crests being viewed from the angles $0^{\circ}, 26.57^{\circ}$ and $45^{\circ}$ respectively to the crests. It is found in the computed data that the crest height diminishes slowly with time with corresponding rise in the trough depth. This feature diminishes with the increase in the angle, becoming insignificant in the third case.

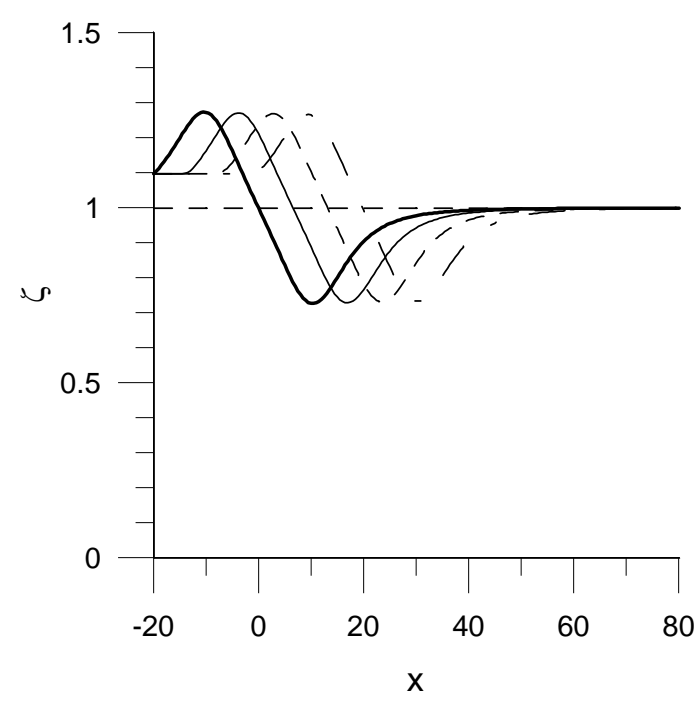

Figure 1. View of propagating surface from $0^{\circ}$ angle, at times $t=0,20,40,60 \ldots$ 


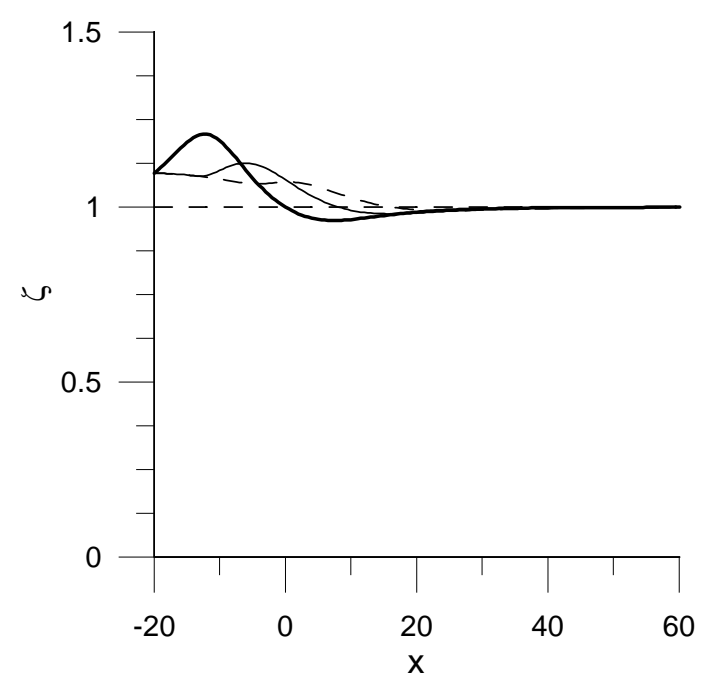

Figure 2. View of propagating surfae from $26.57^{\circ}$ angle, at times $t=0,20,40 \ldots$

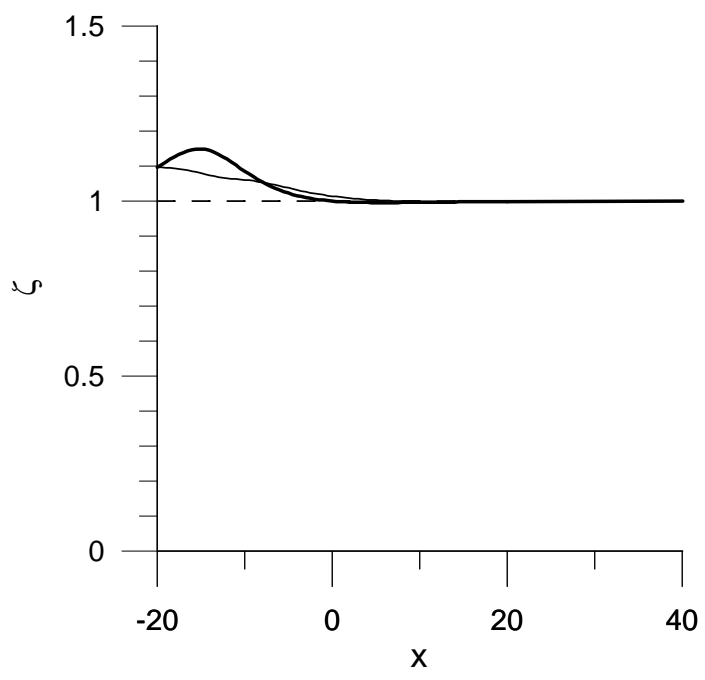

Figure 3. View of propagating surface from $45^{\circ}$ angle at times $t=0,20, \ldots$

The method can be employed to generate data for any other cross section.

As a comparison of the computed results presented above, one may compare these with those of Howe [17], presented on page 333. The surface profiles presented there are based on potential surface wave theory according to which the elevation $\zeta$ is given by the Eq. (5.7.7), p. 330 of the text. The notable qualitative difference between the two approaches is that while in the theory presented here, while the wave form realistically leans forward as it propagates, the form does not replicate this characteristic in the theory presented in Howe [17].

\section{Results and Discussion}

In this paper, the turbulent shallow water free surface flow equations in two dimensions are developed and numerically integrated by a method developed in Bose [14]. Following Bose and Dey [12], the basic equations of continuity and momentum conservation for the flow are developed from the Raynolds Averaged Navier Stokes equations. The equations are expressed in terms of the depth 
averaged horizontal velocity $(U, V)$ and the surface elevation $\zeta$, all being functions of $x, y$ and time $t$. Based on certain assumptions necessitated by the presence of turbulence, three nonlinear differential equations are obtained for the determination of $\zeta, U$ and $V$. For numerical treatment of the equations, a slight simplification is made in the two horizontal momentum equations where in the terms arising from instantaneous vertical acceleration, the quantities $\partial U / \partial t, \partial V / \partial t$ and $\partial U / \partial x+\partial V / \partial y$ are replaced by equivalent expressions in terms of $\zeta$ obtained by linearizing the two equations . A transformation of the equations is then made by replacing $U$ and $V$ by discharge components $Q$ and $R$ respectively. An invertibility argument of the functions shows that $Q$ and $R$ can be functions of $\zeta$, say $Q=F(\zeta)$ and $R=G(\zeta)$, as in the theory of long tidal waves. The method may therefore be considered an extension of the linear inviscid long wave theory to nonlinear waves taking in to account the generated turbulence in real fluids. Substitution in the continuity equation leads to a conservation equation of standard form, while the two momentum equations yield coupled second degree equations in the derivatives $F^{\prime}(\zeta)$ and $G^{\prime}(\zeta)$. In the important case of propagation of nearly linear crested waves on the water surface parallel to $y$-axis, $F^{\prime}(\zeta)$ is given to a first order by a decoupled quadratic equation, and $G^{\prime}(\zeta)$ by an expression containing $F(\zeta)$ and $F^{\prime}(\zeta)$. The two equations are numerically treated to compute $F(\zeta)$ and $G(\zeta)$, following which the standard form mass conservation equation is numerically solved by the Lax-Richtmyer scheme. The method is implemented for an almost linear initial bed elevation model given in Howe [17] for simulating tsunami propagation. The computed values are shown for three different angles of view, showing very slowly decreasing wave height. The method developed in the paper opens up the possibility of generating data for creating animation of the moving free surface for greater visual effect.

\section{Acknowledgement}

The author is thankful to the SN Bose National Center for Basic Sciences, Kolkata for providing necessary facilities for undertaking this research.

\section{References}

[1] C.L. Mader, Numerical Modeling of Water Waves, Boca Raton: CRC Press, 2004.

[2] R.J. Fennema and M.H Chaudhry, "Implicit methods for two-dimensional unsteady free surface flows." J. Hydraul. Res., vol. 27, pp. 321-332, 1989

[3] R.J. Fennema and M.H. Chaudhry, "Explicit methods for 2-D transient free surface flows." J. Hydraul. Engg., vol. 116, pp. 610-614, 1990.

[4] P. Wilders, Th. L.van Stijn, G.S. Stelling, and G.A. Fokkema, "A fully implicit splitting method for accurate tidal computation." Int. J. Num. Meth. Engg., vol. 26, pp. 2707-2721, 1988.

[5] V. Casulli, " Semi-implicit finite difference methods for two-dimensional shallow water equations." J. Comput. Phys., vol. 86, pp. 56-74, 1990.

[6] C. Ouyang, S. He, Q. Xu, Y. Luo and W. Zhang, W., "A MacCormack-TVD finite difference method to simulate the mass flow in mountainous terrain with variable computational domain." Computers Geosci., vol. 52, pp. 1-10, 2013.

[7] S. Fu and B.R. Hodges, Numerical solution of the shallow water equations using a time-centered split-implicit method." 18th Engg. Mech. Div. Confce. (EMD2007), pp. 1-6, 2007.

[8] N.D. Katopodes and T. Strelkoff, " Twodimensional shallow water-wave models." ASCE J. Engg. Mech. Div., vol. 105, pp. 317-334, 1979.

[9] F. Alcrudo and P. Garcia-Navarro, "A highresolution Godunove-type scheme in finite volumes for the 2D shallow-water equations." Int. J. Num. Meth. in Fluids, vol. 16, pp. 489-505, 1993.

[10] S-H. Peng and C. Tang, " Development and application of two-dimensional numerical model on shallow water flows using finite volume method," J. App. Math. Phys., vol. 3, pp. 989-996, 2015. 
[11] P. Garcia-Navarro, M.E Hubbard and A. free surface flow equations." Comput. Geosci., vol. Priestley, "Genuinely multidimensional upwinding 22, pp. 81-86, 2018.

for the 2D shallow water equations." J. Comput. Phys., vol. 121, pp. 79-93, 1995.

[15] S.K. Bose, Numerical Methods of Mathematics Implemented in Fortran, Singapore: Springer, [12] S.K. Bose and S. Dey, "Curvilinear flow pro- 2019.

files based on Reynolds averaging. " J. Hydraul. Engg., vol. 133, pp. 1074-1079, 2007.

[16] R.D. Richtmyer and K.W. Morton, Difference Methods for Initial Value Problems, New York: In[13] H. Sclichting, Boundary Layer Theory, Berlim: terscience, 1967.

Springer, 1979.

[14] S.K. Bose, " A numerical method for the solution of the nonlinear turbulent one-dimensional

[17] M.S. Howe, Hydrodynamics and Sound, Cambridge: Camb. Univ. Press, 2007.

\section{Creative Commons Attribution License 4.0 (Attribution 4.0 International, CC BY 4.0)}

This article is published under the terms of the Creative Commons Attribution License 4.0

https://creativecommons.org/licenses/by/4.0/deed.en_US 